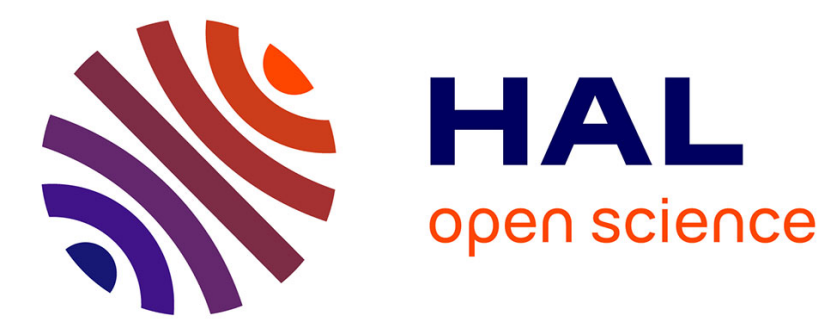

\title{
RESONANCE MODEL FOR PAIR CORRELATIONS IN HEAVY FERMION SYSTEMS
}

\author{
K. Matho, C. Marcenat
}

\section{To cite this version:}

K. Matho, C. Marcenat. RESONANCE MODEL FOR PAIR CORRELATIONS IN HEAVY FERMION SYSTEMS. Journal de Physique Colloques, 1988, 49 (C8), pp.C8-717-C8-718. 10.1051/jphyscol:19888325 . jpa-00228500

\section{HAL Id: jpa-00228500 https://hal.science/jpa-00228500}

Submitted on 1 Jan 1988

HAL is a multi-disciplinary open access archive for the deposit and dissemination of scientific research documents, whether they are published or not. The documents may come from teaching and research institutions in France or abroad, or from public or private research centers.
L'archive ouverte pluridisciplinaire HAL, est destinée au dépôt et à la diffusion de documents scientifiques de niveau recherche, publiés ou non, émanant des établissements d'enseignement et de recherche français ou étrangers, des laboratoires publics ou privés. 


\title{
RESONANCE MODEL FOR PAIR CORRELATIONS IN HEAVY FERMION SYSTEMS
}

\author{
K. Matho and C. Marcenat ${ }^{1}$ \\ C.R.T.B.T., C.N.R.S., 38042 Grenoble Cedex, France
}

Abstract. - The free energy and static spin-correlation function of a two site resonance model akin to the single site Schotte model are calculated. Thermodynamic functions are discussed and model parameters are linked to those of the microscopic two site Kondo model.

\section{Introduction}

Single site resonance (SSR-) models $[1,2]$ are employed to generate analytical expressions for the thermodynamic functions of HF metals. The unique energy scale $\Delta=k_{B} T_{\mathrm{K}_{0}}$ appearing in these models represents the strong coupling scale of the microscopic Kondo impurity problem. Since the exact solution of the latter (as far as free energy is concerned) the excellent semi-quantitative virtues of this SSRphenomenology have become even more evident.

We introduce, at present, the simplest two site resonance (TSR-) model in order to cope with short ranged interactions in the paramagnetic phase of HF compounds. The competition between a magnetic order parameter and Kondo effect has already been treated within SSR + molecular field [3]. In contrast to this, the TSR-model incorporates interaction without breaking the symmetry.

The Hamiltonian is

$$
-\beta \mathcal{H}=\omega \mathbf{S}_{1} \mathbf{S}_{2}+\varepsilon\left(S_{1}^{z}+S_{2}^{z}\right), \quad \beta=\left(k_{\mathrm{B}} T\right)^{-1} .
$$

A Heisenberg interaction $W=\omega / \beta$ competes with a resonance $\rho(\varepsilon)$ in the total angular momentum channel $\mathbf{j}=\mathbf{S}_{1}+\mathbf{S}_{2}$ of two quantum spins $S=1 / 2$. The resonant distribution $\rho(\varepsilon)=\pi^{-1} \mathrm{~d} \delta(\varepsilon / \beta) / \mathrm{d} \varepsilon$ is derived from a phase shift $\delta(E)$, the energy dependence of which is:

$$
\begin{aligned}
& \delta(E)= \\
& =(D \operatorname{arcctg}(-E / \Delta)-\Delta \operatorname{arcctg}(-E / D)) /(D-\Delta)
\end{aligned}
$$

Here, $\Delta=k_{\mathrm{B}} T_{\mathrm{K}}$ is the above mentioned Kondo scale, renormalized by the Heisenberg interaction (see discussion); and $D \gg \Delta$ is a cut-off energy, causing the resonance to be essentially Lorentzian.

Like in the Schotte model [1] we include an applied field $H=h /(\beta \mu)$ by shifting the resonance, assuming $\rho(\varepsilon, h)=\pi^{-1} \mathrm{~d} \delta((\varepsilon-h) / \beta) / \mathrm{d} \varepsilon$.

\footnotetext{
${ }^{1}$ DPCM, Section Physique, CH-1211 Geneva, Switzerland.
}

\section{Free energy and thermodynamics}

The free energy $F(T, H)$ for our model is defined as $F=-k_{\mathrm{B}} T\langle\ln S p \exp (-\beta \mathcal{H})\rangle, S p$ indicating the trace operation and $\langle\ldots\rangle$ the average over $\rho(\varepsilon, h)$. Writing $\phi=\beta F+\omega / 4+\ln \left(4 \pi^{2}\right)$, the result is given in terms of the complex gamma-function:

$$
\begin{gathered}
\phi=2 \sum_{l=1,2} \sum_{\sigma= \pm} c_{l} \operatorname{Re} \Gamma\left(1 / 2-u_{l \sigma}\right) \\
u_{l \sigma}=\left(h+i \beta \Delta_{l}+b_{\sigma}(\omega)\right) / 2 \pi i
\end{gathered}
$$

with $\Delta_{1}=\Delta, \Delta_{2}=D$ and $c_{1}=1-c_{2}=D /(D-\Delta)$. The functions $b_{+}(\omega)$ and $b_{-}(\omega)$ are the two solutions of:

$$
b_{\sigma}(\omega)=\operatorname{arccosh}((1+\exp (-\omega)) / 2)
$$

which obey $b_{-}(\omega)=-b_{+}(\omega)$. They are purely imaginary and bounded, $(|b| \leq \pi / 3)$, in the ferromagnetic (f) case, $\omega>0$, as opposed to purely real and unbounded in the antiferromagnetic (af) case, $\omega<0$.

In the following, we have evaluated some of the thermodynamic derivatives, for $D / \Delta=10^{3}$. The magnetic susceptibility $\chi=\mathrm{d} M / \mathrm{d} H=-\beta \mu^{2} \mathrm{~d}^{2} \phi / \mathrm{d} h^{2}$ is shown in figure 1. The resonant behaviour of $\chi(H)$, accompanied by a flat maximum in the initiai $\chi_{0}(T)$ (not shown) occurs for (af) interactions $-\sqrt{3} W>k_{\mathrm{B}} T_{\mathrm{K}}$. The peak in $\chi(H)$ is attenuated with rising temperature. The threshold field $H_{W}$, defined by $\chi_{\max }(H)=$ $\chi\left(H_{W}\right)$, is practically independent of $T$ and first rises non linearly with $|W|$ before tending to $H_{W} \simeq-W / \mu$

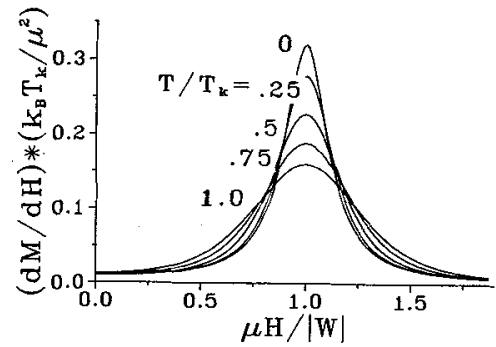

Fig. 1. - Susceptibility $\chi(H)$ in units of $\mu^{2} / k_{\mathrm{B}} T_{\mathrm{K}}$. Parameter $-W / k_{\mathrm{B}} T_{\mathrm{K}}=8$. 
in the limit of strong (af)- coupling. In fact, since $b_{\sigma}(\omega) \simeq-\sigma \omega$ in this limit, (cf. Eq. (4)), the TSR-model asymptotically reduces to two independent SSR- resonances with shifted spectra $\rho(\varepsilon \pm \omega)$. Such shifts have previously been introduced ad-hoc into SSR-models [4], usually in order to generate experimental features that are ascribed to the "coherence" between Kondo sites. Here, they are a consequence of an (af) interaction between sites.

The $T$-derivative of the entropy, $\gamma(T)=C / T=$ $-k_{\mathrm{B}}^{2} \beta^{3} d^{2} \phi / \mathrm{d} \beta^{2}$ is plotted in the next figure. Choosing $\sqrt{3} \mu H=k_{\mathrm{B}} T_{\mathrm{K}}$ for the external field, the usual $T^{2}$-term in $\gamma(T)$ for $W=0$ happens to cancel and the drastic effect of even small Heisenberg couplings on the entropy can be displayed more clearly. In fact, the limit of $\gamma(T=0)$ for $W \rightarrow 0_{+}$is different from the non interacting system; $\gamma(0)$ as a function of $W / k_{\mathrm{B}} T_{\mathrm{K}}$ is discontinuous at this point. At rising $T, \gamma(T)$ goes through step like or bell-shaped resonances in the (f)and (af)-cases, respectively, with characteristics $T$ 's of width and position $\sim|W| /\left(\pi k_{\mathrm{B}}\right)$, before reaching the non-inteacting $\gamma$. Note that the surface under each curve $\gamma(T)$, integrated to $T=\infty$ is $\ln 4$, corresponding to the total entropy.

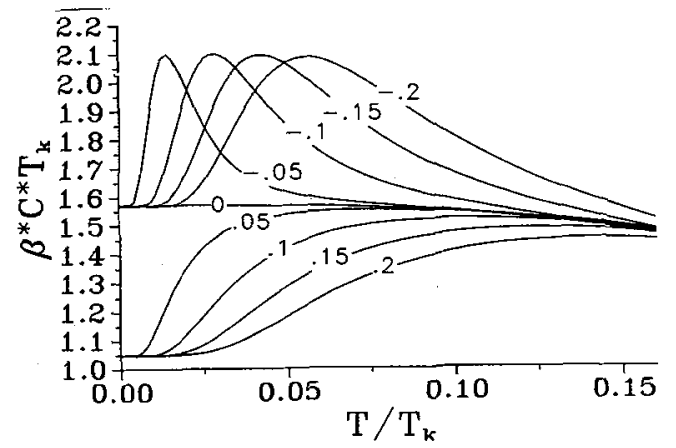

Fig. 2. - Entropy derivative $\gamma(T)=C / T$ in units of $k_{\mathrm{B}} / T_{\mathrm{K}}$. Parameters $W / k_{\mathrm{B}} T_{\mathrm{K}}$ as label, $\mu H / k_{\mathrm{B}} T_{\mathrm{K}}=0.577$.

The static spin-correlation function

$$
\left\langle\mathbf{S}_{1} \mathbf{S}_{2}\right\rangle=-\mathrm{d} F / \mathrm{d} W=\frac{1}{4}-\mathrm{d} \phi / \mathrm{d} \omega
$$

is displayed for $H=0$ in figure 3 . The $W=0$ curve shows spontaneous (f)-like correlations below $T_{\mathrm{K}}$ which are due to the assumption of a coherent resonance in the channel of total angular momentum $\overrightarrow{\mathbf{j}}$. These are enhanced (reduced) by (f)- (resp. (af)) couplings. In a small interval $-0.6 \mathrm{~W} / k_{\mathrm{B}} T_{\mathrm{K}}-0.4$, the correlations may change sign as a function of $T$, (af)- like at low $T$ and (f)- like at high $T$. The $T=0$ correlations are $\equiv 1 / 4$ for (f)- coupling, decrease with rising (af)coupling and saturate at $-3 / 4$ for $W / k_{\mathrm{B}} T_{\mathrm{K}} \rightarrow-\infty$.

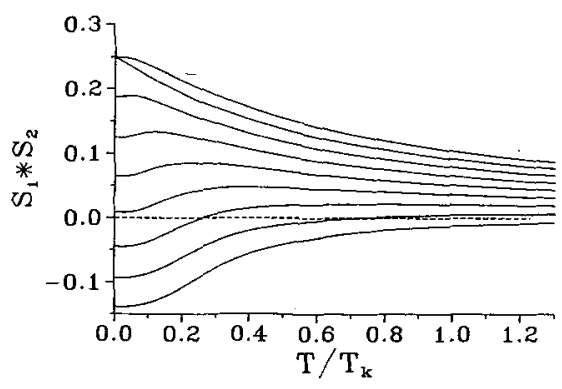

Fig. 3. - Correlation function $\left\langle\mathbf{S}_{1} \mathbf{S}_{2}\right\rangle=-\mathrm{d} F / \mathrm{dW}$. A dependence of $T_{\mathrm{K}}$ on $W$ is not taken into account here. Parameter: $-0.7 \leq W / k_{\mathrm{B}} T_{\mathrm{K}} \leq+0.1$, step 0.1.

\section{Discussion}

We have assumed here that a coherent resonance in the total angular momentum, rather than incoherent resonances for each site, constitutes the appropriate TSR- model for dense HF systems. This phenomenological choice is backed up by the fact that the two site Kondo effect arises in a channel of the $T$-matrix that is proportional to $\vec{j}^{2}$, [5]. In order to renormalize the resonance width $\Delta=k_{\mathrm{B}} T_{\mathrm{K}}$, as a function of the bare interaction. $W_{\mathrm{RKKY}}$, the perturbation theoretical results for $T_{\mathrm{K}}\left(W_{\mathrm{RKKY}}\right)$, [5], can be used. They have been essentially confirmed by the "poor man's scaling" approach [6]. Wether $W$ itself is renormalized, in particular when initially $\left|W_{\mathrm{RKKY}}\right| \ll k_{\mathrm{B}} T_{\mathrm{K}_{0}}$, is still a question presently debated [7, 8]. Extensions of the TSR-model to anisotropic interactions are now being studied.

[1] Schotte, K. D. and Schotte, U., Phys. Lett. A 55 (1975) 38.

[2] Rajan, V. T., Phys. Rev. Lett. 51 (1983) 308.

[3] Bredl, C. D., Steglich, F. and Schotte, K. D., $Z$. Phys. B 29 (1978) 327.

[4] Flouquet, J., Haen, P., Marcenat, C., Lejay, P., Amato, A., Jaccard, D. and Walker, E., J. Magn. Magn. Mater. (1985) 85.

[5] Matho, K. and Beal-Monod, M. T., J. Phys. F 3 (1973) 136.

[6] Jayaprakash, C., Krishna-Murthy, H. R. and Wilkins, J. W., Phys. Rev. Lett. 47 (1981) 737.

[7] Jones, B. A. and Varma, C. M., Phys. Rev. Lett. 58 (1987) 843.

[8] Fye, R. M., Hirsch, J. E. and Scalapino, D. J., Phys. Rev. B 35 (1987) 4901. 